\title{
Coalition Formation in Transmission Expansion Planning
}

\author{
Javier Contreras, Member, IEEE \\ Instituto de Investigación Tecnológica \\ Escuela Técnica Superior de Ingeniería (ICAI) \\ Universidad Pontificia Comillas \\ 28015 Madrid, Spain
}

\author{
Felix F. Wu, Fellow, IEEE \\ Department of Electrical and Electronic Engineering \\ University of Hong Kong \\ Hong Kong \\ Department of Electrical Engineering and Computer Sciences \\ University of California at Berkeley \\ Berkeley, CA 94720, USA
}

\begin{abstract}
The study of a decentralized coalition formation scheme in a specific power systems transmission expansion scenario is the purpose of this paper. We define first who are the agents in the expansion game and provide a decentralized coalition scheme based on Bilateral Shapley values. Finally, we allocate the total costs of expansion amongst the agents, based on the coalition history, and we compare our method with a centralized scheme.
\end{abstract}

Keywords: Transmission planning, power systems, cooperative game theory, multi-agent systems

\section{INTRODUCTION}

Power systems transmission planning addresses the problem of determining the optimal number of lines that should be added to an existing network to supply the forecasted load as economically as possible, subject to operating constraints. "The objective is the minimum cost expansion plan given the base network configuration, the generation facilities, and the forecasted demands for a target year [1]."

Traditionally, the transmission expansion planning problem has been studied using two types of techniques: (1) techniques based on mathematical programming, such as Branch-and-Bound $[2,3,4]$, and techniques based on sensitivity analysis $[5,6]$. There is a third technique that uses neural networks hybridized with genetic algorithms [7], that has shown good performance. The combinatorial nature of the problem made a formidable task to pursue optimal solutions, making it very hard to find reasonable

PE-116-PWRS-0-08-1998 A paper recommended and approved by the IEEE Power System Planning and Implementation Committee of the IEEE Power Engineering Society for publication in the IEEE Transactions on Power Systems. Manuscript submitted November 3 , 1997; made available for printing August 14, 1998. solutions in short computational time.

Since the initial PURPA regulations starting in 1992, the electric utility industry has been facing deregulation to allow transmission open access for suppliers and customers. In the near future, transmission expansion planning will involve decisions taken by some of the actors in the expansion scenario (suppliers, customers, and/or transmission line owners) that can and will affect decisions taken by other players. This intertwined decision process occurs when a new transmission line is built, and it is shared by several "players" of the expansion game. The decision whether to build the line or not, and the allocation of costs to the players who will use the line is still an open issue in

Game theory (GT) is sometimes described as multiperson decision theory or the analysis of conflict. One of the applications of GT has been the modeling of sustaining cooperation in apparently noncooperative environments through repeated interactions. As a modeling tool, cooperative GT has been successfully applied in the power systems area to share the gains of regional cooperation in centralized planning investments [8], to allocate wheeling transaction costs [9], or to allocate cost savings in an energy brokerage system [10].

Research on Distributed Artificial Intelligence (DAI) has focused on how coalitions are formed and on negotiation algorithms amongst players of economic games. Until now, the range of applications of DAI has been restricted to other fields not related to power systems planning: stock market trading [11], cooperative databases [12], and algorithmic theory [14]. Cooperative game theory concepts have been used, but they were suited to decentralized multitask environments $[11,12,13,14,15]$.

Given all that, we propose a combined GT/DAI approach to transmission planning that addresses and solves the pending issues of transmission expansion in a deregulated electricity industry [21]:

- Determining how coalitions are formed

- Implementing a negotiation algorithm a decentralized environment. 
- Allocating total expansion costs to every single agent of the transmission game

These three issues are addressed in a deregulated environment by creating a multi-agent system, where the players cooperate with each other to achieve the optimal common expansion goal. Here the agents have to fulfill certain number of tasks, like adding new lines, and they want to cooperate forming coalitions to reduce overall costs. Each agent is rational, in the sense of being a utility maximizer, and is "an independently motivated agent, not willing to settle for a plan generated by a centralized planner [11]."

\section{NETWORK EXPANSION MODEL}

The objective of the mathematical model of a transmission expansion planning problem is minimizing the capital and operating costs associated with the system expansion over the planning horizon. The constraints associated with this model are the physical and economical constraints that are important when attempting to expand a utility system at a minimum cost, and yet meet all economic and demand restrictions that are placed upon the system. We can formulate the transmission expansion planning problem in the following terms:

$$
\min \sum_{i \in N_{g}} C_{i} P_{G_{i}}+\sum_{j \in A_{n}} K_{j}\left(Z_{j}-Z_{j}^{0}\right)
$$

where $C_{i}$ is the cost per unit power at node $i$ for the whole planning period, $P_{G_{i}}$ is the real power injected into the network by generators at node $i, K_{j}$ is the construction investment cost per parallel link of line $j, Z_{j}$ is the variable representing the total number of parallel links of line $j, Z_{j}^{0}$ is the initial number of parallel links of line $j$, $N_{g}$ is the set of generator nodes, and $A_{n}$ is the set of possible lines.

Assuming a DC load flow model for simplicity, the problem is subject to the following constraints:

(a) Power nodal balance at each bus, Kirchhoff's laws:

$$
A \cdot T=P
$$

where $A$ is the node-branch incidence matrix, $T$ is the branch power flows vector, and $P$ is the net power injections vector.

(b) Limits in branch power flow:

$$
|T| \leq T_{\max }\left(Z_{j}\right)
$$

where $T_{\max }$ is the branch power flow limit vector.

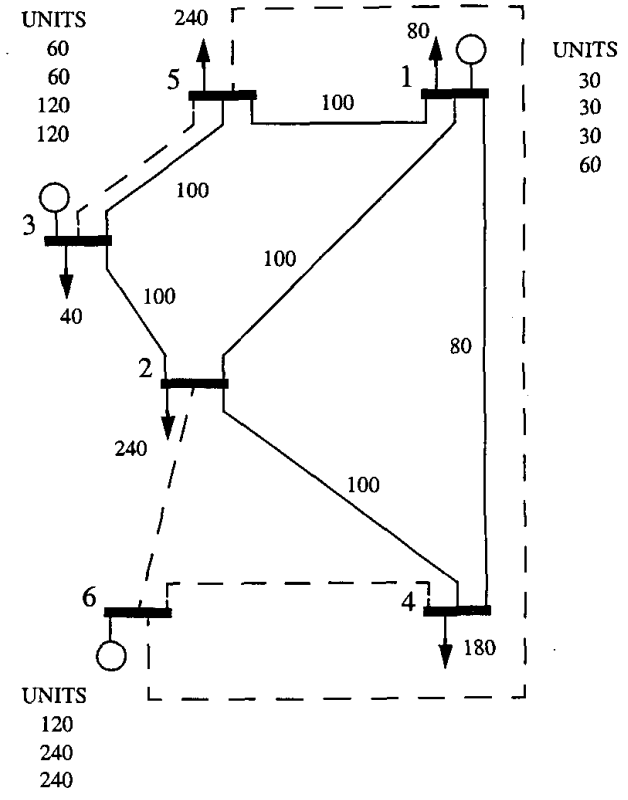

Fig. 1. 6 Bus Problem

In a DC load flow model, each element of the branch power flow vector $T$ in constraint (2) can be described as follows:

$$
T_{j}=\frac{Z_{j}}{x_{j}}\left(\theta_{j}-\theta_{l}\right)
$$

where $Z_{j}$ is the variable representing the total number of parallel links of line $j, x_{j}$ is the reactance of a link of branch $j$ and $\theta_{j}$ and $\theta_{l}$ are the voltages angles of the terminal buses of branch $j$. Then, constraint (2) becomes:

$$
B\left(Z_{j}\right) \cdot \Theta=P
$$

where $B\left(Z_{j}\right)$ is the susceptance matrix whose elements are: $B_{k l}=-1 / x_{k l}$ for the off-diagonal terms, and $B_{k k}=$ sum for all $l$ of $B_{k l}$ susceptances for the diagonal terms, $x_{k l}$ is the total reactance of branch $(k, l), l \in \Omega_{k}$ are the branches connected to bus $k$, and $\Theta$ is the vector of nodal voltage angles. Finally, constraint (3) becomes:

$$
\left|B_{L} A^{t} \Theta\right| \leq T_{\max }\left(Z_{j}\right)
$$

where $B_{L}$ is a diagonal matrix whose elements are: $Z_{j} / x_{j}$.

Let us illustrate the problem formulation by introducing the classical 6 bus system described by Garver in [2], as shown in Figure 1. We will use this simple example in the 


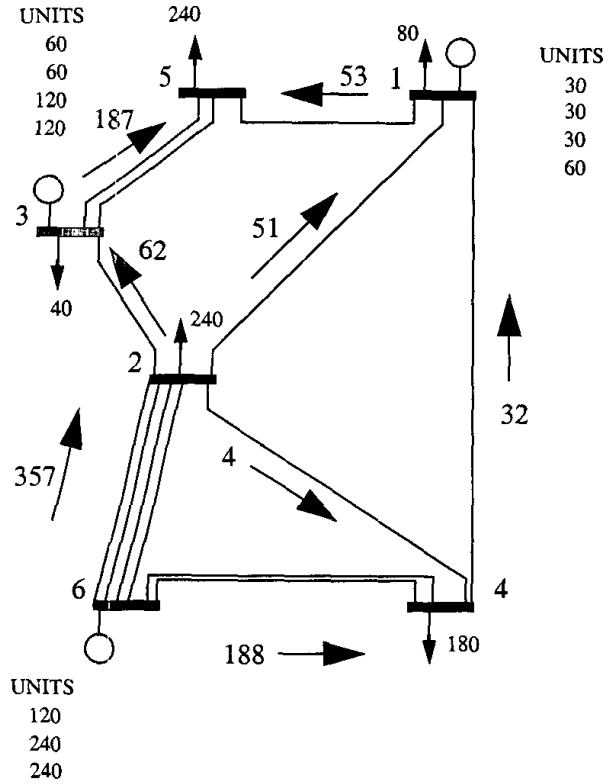

Fig. 2. 6 Bus Example Solution

Table 1: 6 Bus Test System Circuit Data

\begin{tabular}{|c|c|c|c|}
\hline $\begin{array}{c}\text { Bus: } \\
\text { from/to }\end{array}$ & $\begin{array}{c}\text { Cost } \\
\text { (units) }\end{array}$ & $\begin{array}{c}\text { Suscept. } \\
(1 / \Omega)\end{array}$ & $\begin{array}{c}\text { Capacity } \\
(\mathrm{MW})\end{array}$ \\
\hline $1 / 2$ & 40 & 2.50 & 100 \\
\hline $1 / 4$ & 60 & 1.67 & 80 \\
\hline $1 / 5$ & 20 & 5.00 & 100 \\
\hline $2 / 3$ & 20 & 5.00 & 100 \\
\hline $2 / 4$ & 40 & 2.50 & 100 \\
\hline $2 / 6$ & 30 & 3.33 & 100 \\
\hline $3 / 5$ & 20 & 5.00 & 100 \\
\hline $4 / 6$ & 30 & 3.33 & 100 \\
\hline $5 / 6$ & 61 & 1.64 & 78 \\
\hline
\end{tabular}

following sections to simulate coalition formation schemes and to allocate total expansion costs.

We assume that transmission line cost is 1 monetary unit/mile. Its initial configuration has 5 nodes: $1,2,3,4$, and 5 , and 6 branches: $1-2,1-4,1-5,2-3,2-4$, and $3-5$, as shown in Figure 1 and Table 1. When the system expands, there is a new bus: bus 6 , and 4 new possible rights-ofway: $2-6,3-5,4-6$ and $5-6$. The connection between any two buses is allowed with a limit of 4 parallel paths in each right-of-way. Data for existing and future lines in the system is given in Table 1 , where the capacity of each line is determined based on thermal limitations and stability requirements.

The solution obtained by Garver in [2] without generation rescheduling (generator outputs 1, 3, and 6 are 50, 165 and $545 \mathrm{MW}$ respectively) is shown in Figure 2. The arrows indicate the value and direction of the active power flow across the line(s). The optimal solution has a cost of 200 monetary units, and circuit additions are: $n_{26}=4$ circuits, $n_{35}=1$ circuit, and $n_{46}=2$ circuits as shown in Figure 2.

Should we allow generation rescheduling, i.e. the real power generation ranging from 0 to the maximum generation available (150, 360, and $600 \mathrm{MW}$ respectively), the optimal solution had a cost of 130 monetary units, and circuit additions are: $n_{26}=3$ circuits, and $n_{35}=2$ circuits.

\section{COALITIONS IN EXPANSION PLANNING}

In this section we are going to define what is the "game" that is played in a transmission expansion process (from a cooperative game theory standpoint), who are the players (agents ${ }^{1}$ ), and what is a coalition in transmission expansion planning.

The purpose of the game is the expansion of the transmission network, with the minimum possible cost as in (1), subject to constraints given in (2), and (3), and with a "fair" allocation of the total cost among the agents.

An agent in the game can be either a generator, a load, or an independent third party (for example, an independent company who owns transmission lines) that is physically attached to a bus. A typical agent in this context is regarded as an independent entity: a customer or group of customer loads, a generator or a set of generators, or a combination of both. For simplicity, we do not consider for now fractional bus loads or generators, although these would be the "atomic" agents -the minimal ones. We also assume that any set of generation units and loads attached to the same bus belong to a single agent. For our familiar 6 bus example, we have a maximum of 6 agents, corresponding to the 6 generation/load entities attached to the buses.

A coalition of agents is a set of agents consisting of: at least one generator, one load, and one transmission line. There are three axioms that a coalition has to satisfy:

1. We will follow a Multi-Agent System (MAS) terminology, where players are called agents. 
1.- Generator(s) must meet the demand, i.e., the total generator output in the coalition must be greater than or equal to the load.

2.- New and existing line(s) thermal limits can not be exceeded when running a power flow for the new coalition.

3.- There must be one or more transmission lines, either existing or possible candidates, connecting all the buses in the coalition.

These three axioms create what we call autonomous coalitions, because they can try their own expansion plans without having to necessarily negotiate with any other similar entity. Figure 3 shows some examples of feasible autonomous coalitions for the 6 bus case. It can be anticipated from the set of axioms that some of the possible coalitions may be ruled out. In particular, if there is a single bus in the system, with either a generator, a load, or both, it can not be considered a coalition, because it lacks at least one transmission line. Nevertheless, we will assume that single agents will pay for line investments if necessary, so that it is not necessary for them to own transmission lines at the beginning of the expansion game. Also, if two buses meet the first axiom, and there are no line candidates that can connect them, the second axiom is violated. Finally, if not all buses are in the coalition connected to each other, the third axiom is violated.

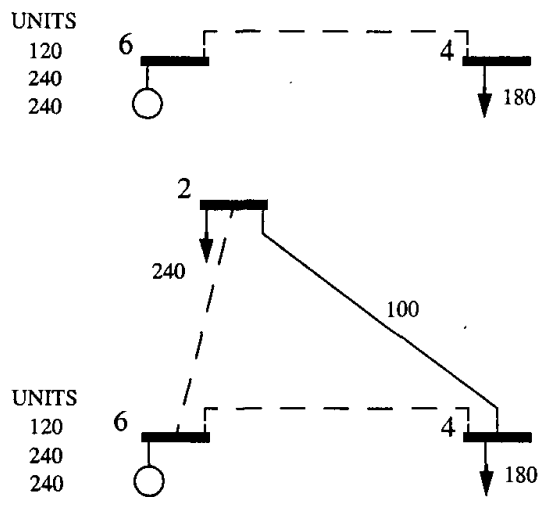

Fig. 3. Examples of "autonomous" coalitions

For the 6 bus case, the set of feasible autonomous coalitions is as follows:

1 agent: $\{2\},\{4\},\{5\}$, and $\{6\}$

2 agents: $\{2,6\},\{3,5\},\{4,6\}$, and $\{5,6\}$

3 agents: $\{1,2,6\},\{1,3,5\},\{1,4,6\},\{1,5,6\}$, $\{2,3,6\},\{2,4,6\},\{2,5,6\},\{3,5,6\}$, and $\{4,5,6\}$

4 agents: $\{1,2,3,6\},\{1,2,4,6\},\{1,2,5,6\},\{1,4,5,6\}$, $\{2,3,4,6\},\{2,3,5,6\}$, and $\{3,4,5,6\}$
5 agents: $\{1,2,3,4,6\},\{1,2,3,5,6\},\{1,2,4,5,6\}$, and

$\{2,3,4,5,6\}$

grand coalition: $\{1,2,3,4,5,6\}$

The other possible coalitions violate at least one of the axioms.

\section{DECENTRALIZED COALITION FORMATION BETWEEN TRANSMISSION EXPANSION AGENTS}

The Shapley value [16] is a cooperative game theory solution concept that calculates a fair division of a common utility (money, resources, etc.) among the members of a coalition. It can be defined as the weighted average of marginal contributions of a member to all possible coalitions in which it may participate. It assumes that the game is superadditive, and that the grand coalition is formed. The mathematical expression of the Shapley value, $\phi_{i}$, is given as follows:

$$
\phi_{i}=\frac{1}{n} \sum_{q=1}^{n} \frac{1}{c(s)} \sum_{i \in s}[v(s)-v(s-i)]
$$

where

$i=$ player

$s=$ coalition of players

$q=$ size of a coalition

$n=$ total number of players

$v(q)=$ characteristic function (cost savings) associated with coalition $q$.

$c(q)=$ number of coalitions of size $q$ containing the designated player $i$, given by,

$$
c(q)=\frac{(n-1) !}{(n-q) !(q-1) !}
$$

In order to avoid the exponential complexity of a Shapley value calculation, Ketchpel introduced the so-called Bilateral Shapley Value (BSV) $[11]^{2}$. Klusch and Shehory $[12,13]$ adapted this approach for a completely decentralized and bilateral negotiation process among rational information agents using these values. In particular, the algorithm for coalition formation that they provided is also useful in the power transmission planning environment. Thus, to formulate our problem using BSVs, let us define our framework first.

2. Similar work has been done by Kraus and Shehory [17]. In contrast to our work, they did not provide a solution for cooperative games and a given coalition structure with other than 2-agents coalitions. 
Let $C S \subseteq P(A)$ be a coalition structure on a given set of agents $A=\left\{a_{1}, \ldots, a_{m}\right\}$, where $C_{i} \cup C_{j} \subseteq A$ is a (bilateral) coalition of disjoint (n-agent) coalitions $C_{i}$ and $C_{j}$ $(n \geq 0)$. The Bilateral Shapley Value for some coalition $C_{i}$ in a bilateral coalition $C$ is defined as $b s v_{\left\{C_{i}, C_{j}\right\}}\left(C_{i}\right):=0.5 v\left(C_{i}\right)+0.5\left(v(C)-v\left(C_{j}\right)\right)$. Both coalitions $C_{i}$ and $C_{j}$ are called founders of $C$, and $v(C)$ clenotes the self-value of coalition $C^{3}$.

It can be seen that the founders will get half of their local contribution, and the other half stemming from cooperative work with the other entity. The second term of the BSV expression reflects the strength of each agent concerning his contribution, therefore avoiding the "freerider" problem, so common in transmission expansion value allocation schemes. The BSV is a particular case of the Shapley value concept, because they create a fair distribution of resources among two agents only.

Our coalition formation method is based on the approach followed by Klusch and Shehory in [13]. A summary of the steps in the coalition formation process is given in the following subsections.

\section{A. Self-Calculation Phase}

Each individual agent gathers information to determine its self-cost. Calculation of the self-cost determines the monetary cost of line expansion for individual agents, following the three axioms from the previous section. It is possible that some of the players are unwilling to use new ines on their own, and this fact is reflected by a cost of zero. For the 6 bus example, where each individual agent is attached to a bus, we can observe that agents 1 and 3 are self-sufficient, because the load is met by the existing bus generation. However, agents 2,4 , and 5 need to use extra lines to meet their own demand. Finally, agent 6 needs to be attached to the network, not to become isolated. These facts are reflected in their self-costs. In the case of different initial settings, agents 3 and 5 being a single initial agent for instance, costs would change, reflecting different initial conditions.

\section{B. Communication Phase}

Once each agent has calculated its own self-cost, it is time to determine the joint cost that he will have when cooperating with another agent. Unfortunately, an agent is not necessarily aware of the environment surrounding him, thus a coordinator is needed to gather this information. This is certainly the case of transmission expansion planning, where the entire network is not known completely by

3. Note that $b s v_{\{C, \varnothing\}}(C)=v(C)$, and $v(\varnothing):=0$

4. The "free-rider" concept addresses the issue of new agents that take advantage of the work done by the existing ones without paying them any compensation any player. In order to calculate the BSVs, the agents need to send all their proposed line addition(s) to this central figure, and then, receive the adequate number of new lines for requested coalitions, via messages sent by the coordinator to all of them. It is possible that two agents reach an agreement that is satisfactory to both of them, but detrimental to the security of the system. That is why the role of an independent coordinator is needed to check network reliability and quality of service. Usually, a power flow subject to security constraints should be enough. On the other hand, agents freely exchange self-costs with each other.

\section{BSV Calculation Phase}

Now the agents know their own self-costs, and every possible cost with other coalition partners via Step B. After getting these messages from the coordinator, they proceed to calculate BSVs if teaming with another agent. Then, the agents determine individually rational ${ }^{5}$ lists of preferred agents: ordered list of local agents's BSVs for two-entity coalitions. These lists will change whenever step $\mathrm{C}$ is called again, with new multi-parties players acting as agents ${ }^{6}$.

\section{Bilateral Negotiation Phase}

Each agent looks at the head of his ordered list, and extends an offer to the preferred partner. The offer consists of sending the partner's BSV: the value that he would attain for collaborating with the sender. If it happens that the sender receives also a message from the preferred partner, and they both find it is beneficial to join, they do. They create a multi-parties player that will behave like one agent from then on. Every other agent is also informed, for him to erase the members of the multi-parties players from his own preference list.

The coalition formation process is repeated by all agents and multi-parties players, starting from Step B, until no more coalitions are possible. If no coalition is possible at one particular step, the agents look at the second best partner; if still not possible, to the third, etc., until reaching the end of the list.

There are several features of this negotiation algorithm that are only relevant to expansion planning. First, this is a general (non superadditive) environment, thus the grand coalition will not necessarily be formed. Second, previous negotiation algorithms used a utilitarian coalition building scheme, where the agents have to satisfy their tasks via cooperation, thus increasing their benefits. The utilitarian

5. Individual rationality means that the agent wants to have a new value that is, at least, as good as the one that he could attain alone.

6. This means that the agents in the coalition will vote for a representative agent that will be the spokesperson for this coalition in the future. 
coalition formation in the transmission expansion domain is done in a cost-oriented view ${ }^{7}$.

Note that even when common resources are shared (the lines), it does not mean that all agents can access all of them. In the case of multi-parties players, where load at bus 2 and generation at bus 6 form a single agent, we disregard all lines connecting agent 2-6 to other agents. For single agent expansion plans, the agent pays in full for all the lines that it needs to meet its own load at a minimum cost.

\section{COST ALLOCATION METHOD: THE BACK- WARDS INDUCTION ALGORITHM}

Defining a coalition formation scheme is not enough to solve the transmission expansion game. Even if all agents agree on the final coalition arrangements, the issue of allocating the total cost among them remains yet unsolved. To address this issue we propose a solution $\left(C,\left(x_{1}, \ldots, x_{n}\right)\right)$ for a given cooperative game $(A, v)$ in general environments ${ }^{8}$ using BSVs. Our method is based on a special algorithmic calculation of each agent's utility $x_{i}(i \in\{1, \ldots, m\})$ which is not necessarily $b s v$, if $a_{i} \in C_{k}$, and $C_{k}$ is a founder of $C$ in the coalition structure $C S$. In the following, we will denote coalitions with more than one agent as (multi-parties) players.

To illustrate the algorithm, let us return to our familiar 6 bus example whose coalition costs are given in Table 2 . We will follow a backward induction algorithm method to calculate value (cost) allocations via BSVs.

Starting from the grand coalition ${ }^{9}$, we divide the team into the two founding members: $[\{1-2-3-5-6\},\{4\}]$, and split the total value (cost) of $-130(130)$. Let $b s v_{\{i, j\}}(i)$ be the value allocated to agent $i$ using the Bilateral Shapley Value rationale:

$$
\begin{gathered}
b s v_{\{12356,4\}}(12356)=1 / 2 v(\{12356\})+ \\
1 / 2(v(\{123456\})-v(\{4\})) \\
b s v_{\{12356,4\}}(4)=1 / 2 v(\{4\})+ \\
1 / 2(v(\{123456\})-v(\{12356\}))
\end{gathered}
$$

where $v(i)$ is the value of coalition $i$, as per Table 2, when we reverse signs. Going backwards one more step, we find the following values for the next subdivision of $[\{1-2-3-5-6\}]$ into their founders $[\{1\}]$ and $[\{2-3-5-6\}]$ :

7. Related work can be found in the DAI area for task-oriented domains with cost functions by Rosenschein and Zlotkin.

8. General environments allow for both, superadditive as well as subadditive games. A game $(A, v)$ is superadditive iff $\forall C=C_{1} \cup C_{2} \subseteq P(A): v(C) \geq v\left(C_{1}\right)+v\left(C_{2}\right) ;$ it is subadditive iff it is not superadditive.

9. See Section VI: Simulation Results.

$$
\begin{gathered}
b s v_{\{1,2356\}}(1)=1 / 2 v(\{1\})+ \\
1 / 2\left(b s v_{\{1,2356\}}(12356)-v(\{2356\})\right) \\
b s v_{\{1,2356\}}(2356)=1 / 2 v(\{2356\})+ \\
1 / 2\left(b s v_{\{1,2356\}}(12356)-v(\{1\})\right)
\end{gathered}
$$

The process is followed until the values for individual agents are found: $(12.5,-49.375,10.625,-55,-29.375$, 19.375 ), adding up to -130 total final value. Note that in the second and subsequent steps of the backward algorithm, the total value to split is given by the previous Bilateral Shapley Value, as shown in [11] and [12], by using $b s v_{\{1,2356\}}(12356)$ instead of $v(\{12356\})$. This backward process can be used as a general calculation scheme for every agent utility in its coalition. Figure 4 illustrates the cost allocation process step-by-step.

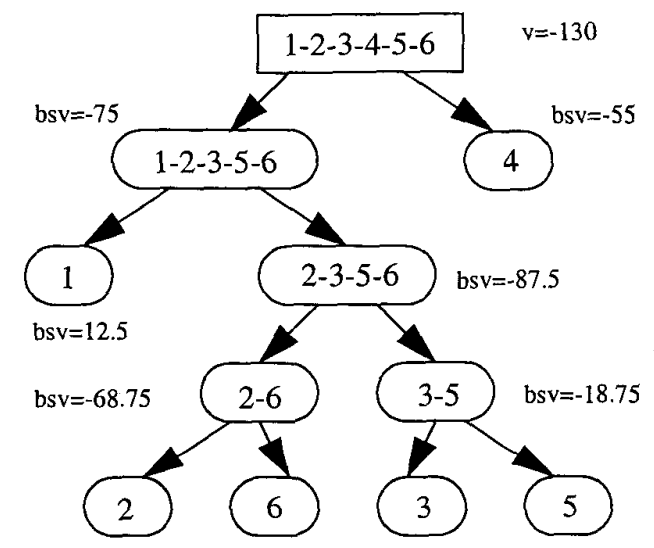

bsv $=-49.375 \quad b s v=-19.375 \quad b s v=10.625 \quad b s v=-29.375$

Fig. 4. Cost allocation results using backwards induction

\section{SIMULATION RESULTS}

We have run a coalition formation simulation for the 6 bus test case implementing the Bilateral Negotiation algorithm. Cost functions are shown in Table 2. Note that when using SVs and BSVs, values are negative to reflect positive expansion costs in monetary units. Note also that the respective coalition values of this (subadditive) game are negative, reflecting the utility of a coalition in a costoriented view ${ }^{10}$.

Table 2: Coalition Expansion Costs

\begin{tabular}{|c|c|c|c|}
\hline Coalition & Cost & Coalition & Cost \\
\hline$\{2\}$ & 90 & $\{2,5,6\}$ & 334 \\
\hline
\end{tabular}

10. E.g., the value (cost) for the grand coalition is $-130(130)$. 


\begin{tabular}{|c|c|c|c|}
\hline Coalition & Cost & Coalition & Cost \\
\hline$\{4\}$ & 60 & $\{3,5,6\}$ & 101 \\
\hline$\{5\}$ & 40 & $\{4,5,6\}$ & 304 \\
\hline$\{6\}$ & 60 & $\{1,2,3,6\}$ & 30 \\
\hline$\{2,6\}$ & 90 & $\{1,2,4,6\}$ & 120 \\
\hline$\{3,5\}$ & 40 & $\{1,2,5,6\}$ & 273 \\
\hline$\{4,6\}$ & 60 & $\{1,4,5,6\}$ & 243 \\
\hline$\{5,6\}$ & 183 & $\{2,3,4,6\}$ & 120 \\
\hline$\{1,2,6\}$ & 60 & $\{2,3,5,6\}$ & 100 \\
\hline$\{1,3,5\}$ & 20 & $\{3,4,5,6\}$ & 161 \\
\hline$\{1,4,6\}$ & 60 & $\{1,2,3,4,6\}$ & 90 \\
\hline$\{1,5,6\}$ & 183 & $\{1,2,3,5,6\}$ & 80 \\
\hline$\{2,3,6\}$ & 60 & $\{1,2,4,5,6\}$ & 272 \\
\hline$\{2,4,6\}$ & 150 & $\{2,3,4,5,6\}$ & 160 \\
\hline & & $\{1,2,3,4,5,6\}$ & 130 \\
\hline & & & \\
\hline
\end{tabular}

The first simulation starts with the individual agents 1 to 6 creating lists of preferences. We assume for the first two simulations that whenever a tie occurs between going solo or teaming, teaming is preferred. The final resulting coalition is $\{1,2,3,4,5,6\}$, and the order of coalition creation is the following: $[1,\{2-6\},\{3-5\}, 4]$ in the first round, $[1$, $\{2-3-5-6\}, 4]$ in the second, $[\{1-2-3-5-6\}, 4]$ in the third, and $[\{1-2-3-4-5-6\}]$ in the fourth and last round. Once the grand coalition is created, we follow a "backwards induction" method to calculate cost allocations via BSvs. Starting from the grand coalition, we divide the team into the two founding members: $[\{1-2-3-5-6\}, 4]$, and split the total cost of 130 in two, using the values from Table 2 . The process is followed until the costs for individual agents are found: $(12.5,-49.375,10.625,-55,-29.375,-$ 19.375), adding up to -130 total.

A second simulation is run, since player 6 is indifferent to team with 2 or 4 at the beginning. The process is now: $[1,2,\{3-5\},\{4-6\}] \rightarrow[\{1-3-5\},\{2-4-6\}] \rightarrow[\{1-2-3-4-5-$ $6\}]$. The cost allocation is given by: $(21.25,-49.375,6.25$, $-55,-33.75,-19.375)$.

Note that not all simulations drive the coalition to a grand coalition scheme. Here is one example: $[1,\{2-6\}, 3$, $4,5] \rightarrow[\{1-2-6\}, 3,4,5] \rightarrow[\{1-2-3-6\}, 4,5]$. But cost allocation is still up to 130 : $(22.5,-48.75,15,-60,-40$,18.75).

Were we using a central planner, we would not need a synchronized and decentralized algorithm. A central coor- dinator would calculate the Shapley values for all agents, given the costs in Table 2 . These should be the allocation results: $(16.85,-45.86,54.73,-19.41,-42.48,-93.81)$. Comparing with the BSV results, we observe a clear mismatch in buses 3 and 6 allocations. The reason is that SVs are only good tools for a superadditive environment where the grand coalition is always formed, which is not the case. SVs consider all possible combinations of players, good and bad. By using BSVs, only the best combinations of players will form, and this will be reflect in the final allocation.

So far, we have considered players that belong to the network. But, in a deregulated environment, we have to consider players that just invest money in building lines, and want to recover that investment. This is the case of third party transmission line owners. For the sake of the example, suppose that line 2-6 belongs to a party that does not own anything else in the network. These are the steps that a decentralized algorithm would follow for the line owner to recover her investment:

1) Determine if the line is beneficial for the entire network. The coordinator runs a transmission line expansion.

2) If the line is beneficial, run the coalition formation algorithm.

3) Recover part, or all investment costs, from the parties that are directly using the line: players 2 and 6 . If they do not provide enough, distribute the remaining costs among the other players in a fair manner (for example, splitting them in equal parts).

For all simulations, buses 1 and 3 receive monetary units for their contribution to the welfare of the system, while the remaining buses must contribute, although less that what they would pay on their own. This is a clear incentive for all players to play this game.

\section{SOFTWARE IMPLEMENTATION}

The network expansion program has been coded in Scilab, a software platform similar to MATLAB $C^{11}$ and developed at INRIA [18].

Mathematica $\left(C^{12}\right.$ is the cost allocation software that has been used for this project. The interested reader is referred to Chapter 8 of [19] for implementation details.

Towards an implementation of Transmission Planning Agents, an Interactive Development Environment for the specification and simulation of Agent Systems IDEAS [12] has been recently implemented on a network of Sun workstations. IDEAS runs under an X-Windows environments

11. MATLAB is a registered trademark of The MathWorks, Inc. 12. Mathematica is a registered trademark of Wolfram Research, Inc. 
and uses Tcl/Tk, C and Prolog to simulate agents' actions, communication and agents' beliefs respectively. At the current time, an implementation of the coalition formation and negotiation process is being tested for the 6 bus system. Due to its modularity, IDEAS is perfectly suitable to be embedded in other software environments, or work as a stand alone program.

\section{CONCLUSIONS}

This paper has presented a new application of decentralized coalition formation and cooperative cost allocation in the power systems transmission planning area. A simple heuristic transmission planning problem has been used to calculate costs for different expansion coalitions. These coalitions have been chosen by introducing two new concepts. First, an "autonomous" coalition set of players has been defined. Second, the concept of "natural" coalition sets has been introduced. A simple 6 bus case has been analyzed using centralized cost allocation techniques, based on SVs, and Distributed Artificial Intelligence (DAI) game theory concepts, like the BSVs. Results on both areas have been presented and analyzed.

We acknowledge that such a simple example is just a first step in the way to understand cost allocation in a decentralized environment. Future work will refine coalition formation schemes, analyze coalition stability, and will explore classical Game Theory concepts, like the kernel, applied to decentralized expansion games.

\section{ACKNOWLEDGMENTS}

The research was supported by Research Grant Council, Hong Kong, under Grant HKU 564/96E, National Science Foundation, U.S.A., under Grant ECS-9527626, and under a Doctors and Technologists Research Grant, Ministerio de Educación y Cultura, Spain.

\section{REFERENCES}

[1] G.C. Oliveira, A.P.C. Costa and S. Binato, "Large Scale Transmission Network Planning Using Optimization and Heuristic Techniques," IEEE Transactions on Power Systems, vol. 10, no. 4, November 1995, pp. 1828-1833.

[2] L.L. Garver, "Transmission Net Estimation Using Linear Programming," IEEE Transactions on Power Apparatus \& Systems, vol. PAS-89, no. 7, Sept/Oct 1970, pp. 1688-1697.

[3] Y.P. Dusonchet and A.H. El-Abiad, "Transmission Planning Using Discrete Dynamic Optimizing," IEEE Transactions on Power Apparatus and Systems, vol. PAS-89, 1973, pp. 1358-1371.

[4] S.T.Y. Lee, K.L. Hocks and E. Hnyilicza, "Transmis- sion Expansion of Branch-And-Bound Integer Programming With Optimal Cost-capacity Curves," IEEE Transactions on Power Apparatus and Systems, vol. PAS-93, no. 5, 1974, pp. 1390-1400.

[5] R.J. Bennon, J.A. Juves and A.P. Meliopoulos, "Use of Sensitivity Analysis Automated Transmission Planning," IEEE Transactions on Power Apparatus and Systems, vol. PAS-101, no. 1, 1982, pp. 53-59.

[6] A. Monticelli, A. Santos Jr., M.V.F. Pereira, S.H. Cunha, B.J. Parker and J.C.G. Praça, "Interactive Transmission Network Planning using a Least-Effort Criterion," IEEE Transactions on Power Apparatus \& Systems, vol. PAS-101, no. 10, October 1982, pp. 3319-3325.

[7] K. Yoshimoto, K. Yasuda and R. Yokohama, "Transmission Expansion Planning Using Neuro-Computing Hybridized with Genetic Algorithm," Proceedings of the 1995 International Conference on Evolutionary Computing, ICEC'95, pp. 126-131.

[8] D. Gately, "Sharing The Gains From Regional Cooperation: A Game Theoretic Application To Planning Investment In Electric Power," International Economic Review, vol. 15, no. 1, February, 1974, pp. 195208.

[9] Y. Tsukamoto and I. Iyoda, "Allocation of Fixed Transmission Cost by Cooperative Game Theory," IEEE Transactions on Power Systems, vol. 11, no. 2, May 1996, pp. 620-629.

[10]D. Chattopadhyay, "An Energy Brokerage System with Emission Trading and Allocation of Cost Savings," IEEE Transactions on Power Systems, vol. 10, no. 4, November 1995, pp. 1939-1945.

[11] S.P. Ketchpel, "Coalition Formation Among Autonomous Agents," Proceedings of MAAMAW-93.

[12] M. Klusch, "Utilitarian coalition formation between information agents for a cooperative discovery of interdatabase dependencies," in S. Kirn and G. O'Hare (Eds.), Cooperative Knowledge Processing, Springer Verlag, London, 1996.

[13] M. Klusch and O. Shehory, "Coalition Formation Among Rational Information Agents," Proceedings of MAAMAW-96, Eindhoven, LNAI Series vol. 1038:204-217, Springer Verlag.

[14] M. Klusch and O. Shehory, "A polynomial kernel-oriented coalition for rational information agents," Proc. 2nd. International Conference on Multi-Agent Systems ICMAS-96, Kyoto (Japan), AAAI Press, 1996.

[15] O. Shehory and S. Kraus, "A kernel-oriented model for coalition formation in general environments: Implementation and results," Proceedings AAAI-96, Portland, OR, 1996.

[16] Shapley, L.S., The Value of an n-person Game, Contributions to the Theory of Games, in H.W. Kuhn and A.W. Tucker, eds., Princeton University Press, 1953. 
[17]O. Shehory and S. Kraus, Coalition Formation Among Autonomous Agents: Strategies and Complexity, Lecture Notes in Artificial Intelligence No. 957, From Reaction to Cognition, C. Castelfranchi and J.P. Muller (Eds.), Springer-Verlag, Berlin, 1993, pp. 5772.

[18] Introduction to Scilab, Scilab Group, INRIA Meta2 Project/ENPC Cergrene, Unité de recherche de Rocquencourt, France, 1996.

[19] Economic and Financial Modeling with Mathematica, Hal R. Varian editor, Telos and Springer-Verlag, Santa Clara, CA, 1993.

[20] Ptolemy Reference Manual, V. 0.7., EECS Department, University of California at Berkeley, 1997.

[21] J. Contreras, A Cooperative Game Theory Approach to Transmission Planning in Power Systems, Ph.D. Thesis, Department of Electrical Engineering and Computer Sciences, University of California at Berkeley, May 1997.

\section{BIOGRAPHIES}

Javier Contreras was born in Zaragoza, Spain, in 1965. He received his B.S. in Electrical Engineering from the University of Zaragoza, Spain, his M.S. from the University of Southern California, and his Ph.D. from the University of California, Berkeley in 1989, 1992, and 1997, respectively. Currently he is a research er at the Instituto de Investigación Tecnológica, Escuela Técnica Superior de Ingeniería (ICAI), Universidad Pontificia Comillas, in Madrid, Spain.

Felix F. Wu received his B.S. from National Taiwan University, his M.S. from the University of Pittsburgh, and his Ph.D. from the University of California, Berkeley. He worked for Pacific Gas and Electric as a Computer Applications Engineer in 1976 77. Dr. Wu was elected a Fellow of the IEEE in 1989. He held a TEPCO (Tokyo Electric Power Company) Professorship at the University of Tokyo in 1991. He is currently Pro Vice Chancellor and Professor of Electrical Engineering at the University of Hong Kong. on leave from UC Berkeley, where he has been a Professor of Electrical Engineering and Computer Sciences since 1982. 